\title{
El proceso de investigación y su aplicación en la rehabilitación respiratoria. Primera parte
}

\section{KIgo. Iván Rodríguez N.}

Escuela de Kinesiología, Facultad de Ciencias de la Salud. Universidad San Sebastián. Concepción. Docente Programa de Magíster en Kinesiología Cardiorrespiratoria, Facultad de Ciencias de la Salud. Universidad San Sebastián. Campus Los Leones. Santiago.

\begin{abstract}
THE RESEARCH PROCESS AND ITS APPLICATION IN PULMONARY REHABILITATION- PART I
In recent years there has been a significant increase in the number of scientific articles published in the rehabilitation field, which has allowed the development of the discipline based on the paradigms of evidence-based medicine. In this sense, to avoid the distortion of the results, caused by systematic or random error, it is crucial to know the methodological elements of the research process because these factors critically affect the validity of the results obtained during clinical research. The present review deals with the initial stages of the research process in an integrated manner. These latter are: the statement of the problem; objectives, hypotheses and study design. The purpose of the review is to provide guidance for the design of future studies in the area of pulmonary rehabilitation.
\end{abstract}

Keywords: biomedical research, clinical research, respiratory therapy, pulmonary rehabilitation.

\section{RESUMEN}

En los últimos años se ha observado un incremento significativo en la cantidad de artículos científicos publicados en el campo de la rehabilitación, lo que ha permitido el desarrollo de la disciplina con un enfoque basado en los paradigmas de la medicina basada en evidencia. En este sentido, conocer los elementos metodológicos que componen el proceso de investigación es fundamental para que los resultados no sufran distorsiones producidas por error sistemático 0 azar, factores que afectan de manera crítica la validez de los resultados obtenidos durante la investigación clínica. En la presente revisión se aborda de manera integrada las fases iniciales del proceso de investigación, que son: el planteamiento del problema; objetivos, hipótesis y diseño de estudio. El propósito es aportar una guía para el diseño de futuros estudios en el área de la rehabilitación pulmonar

Palabras clave: investigación biomédica, investigación clínica, terapia respiratoria, rehabilitación pulmonar.

\section{INTRODUCCIÓN}

En los últimos años hemos sido testigos de un incremento significativo en la cantidad de artículos científicos publicados en el campo de la rehabilitación, lo que ha favorecido la construcción de guías de recomendación que han permitido el desarrollo de la disciplina con un enfoque basado en los paradigmas de la medicina basada en evidencia (MBE) (1-3). Esto ha sido posible gracias a que se ha logrado comprender que los conocimientos acerca de las respuestas y adaptaciones fisiológicas a la rehabilitación no son suficientes para explicar, en su totalidad, el efecto clínico de las estrategias dirigidas al tratamiento y rehabilitación de las diversas entidades nosológicas que afectan el sistema cardiorrespiratorio en el niño (2).

\footnotetext{
Correspondencia

Klgo. Iván Rodríguez Núñez. MSc. PhD

Escuela de Kinesiología

Facultad de Ciencias de la Salud

Universidad San Sebastián. Lientur №1457

Concepción, Chile

Teléfono: 041-2487293

e-mail: ivan.rodriguez@uss.cl
}

ISSN 0718-333X. Derechos reservados.
La veracidad de los resultados de un estudio científico depende de la validez interna del mismo. En este contexto, conocer los elementos metodológicos que componen el proceso de investigación es fundamental para el desarrollo de estrategias que permitan mitigar error sistemático o azar, factores que afectan de manera crítica la validez de los resultados obtenidos.

En la presente revisión se pretende mostrar de manera integrada las etapas iniciales del proceso de investigación y su aplicación en el área de la kinesiología cardiorrespiratoria, con el propósito de aportar una guía para el diseño de futuros estudios en esta área.

\section{PROBLEMA DE INVESTIGACIÓN}

La génesis de todo estudio radica en el establecimiento de un problema de investigación que, a menudo, se expresa en forma de pregunta. No obstante, previo al diseño de la pregunta, es indispensable el desarrollo de una idea, punto de interés, la que debe ser abordada integralmente y desde varias dimensiones. Por ejemplo, "rehabilitación en niños con fibrosis quística", constituye un área temática (punto de interés) que puede ser abordado desde la perspectiva de la evaluación, tratamiento, bases fisiológicas, aspectos sicosociales, etc, sin llegar a constituir una pregunta de investigación posible de ser 
respondida. Por este motivo, profundizar en las características, contexto e importancia del enunciado del problema, permitirá construir una pregunta de investigación robusta, que sea un reflejo claro del diseño de investigación que se debe emplear para responderla (4).

La pregunta de investigación debe ser construida en función del criterio "PICoR" (Pacientes, Intervención, Comparación y Resultados) y debe ser expresada en términos interrogativos (no declarativos) $(5,6)$. Un ejemplo de pregunta de investigación para el tema de interés "rehabilitación en niños con fibrosis quística" puede ser: ¿Cuál es la mejor estrategia de rehabilitación entre entrenamiento aeróbico (intervención) y entrenamiento anaeróbico asistido con ventilación no invasiva (comparación) en niños con fibrosis quística (pacientes), en términos de consumo de oxígeno, trabajo máximo, calidad de vida y sobrevida (resultados)?

Queda claro que el escenario en el cual está inmerso el problema de investigación citado precedentemente corresponde al de terapia, prevención, etiología y daño; por lo tanto, una revisión sistemática con metanálisis de ensayos clínicos con homogeneidad o un ensayo clínico con asignación aleatoria e intervalos de confianza estrechos, constituyen los tipos de diseños que proporcionan el mejor nivel de evidencia para responder a esta pregunta (evidencia tipo 1a y $1 \mathrm{~b}$ respectivamente) (7).

Otro punto que debe ser expresado en esta etapa del proceso de investigación corresponde a la justificación del estudio, el cual debe ser abordado considerando el vacío de conocimiento existente, el propósito que motiva la realización del estudio, la importancia que trae consigo la adquisición del nuevo conocimiento y la factibilidad de la realización del protocolo de investigación. En el caso del problema de investigación planteado en los párrafos anteriores, el vacío de conocimiento radica en que son desconocidos los efectos del ejercicio asistido con VNI en este grupo de pacientes; la importancia de desarrollar el estudio radica en que nos permitirá conocer los efectos fisiológicos a corto y largo plazo que produciría la incorporación de VNI a los programas de rehabilitación, y el propósito de estudio sería proporcionar antecedentes que permitan la incorporación de esta estrategia de rehabilitación a los protocolos recomendados por los estamentos oficiales (8).

\section{OBJETIVOS}

El enunciar el problema de investigación en forma de pregunta nos induce a la necesidad de generar una respuesta para resolverla y probar la teoría en cuestión. En este contexto, el planteamiento de objetivos permite expresar el motivo general de la investigación, este debe ser redactado en forma declarativa donde se establezca el rumbo general de la investigación y proporcione una sinopsis de su meta global. En los estudios cuantitativos el enunciado del objetivo general debe ser construido siguiendo el criterio PICoR. A modo de ejemplo, el objetivo general del estudio citado precedentemente podría ser
"Evaluar el efecto del entrenamiento aeróbico comparado con entrenamiento anaeróbico asistido con ventilación no invasiva en términos de consumo de oxígeno, trabajo máximo, calidad de vida y sobrevida, en niños con fibrosis quística" (4).

\section{HIPÓTESIS}

La hipótesis de investigación constituye un intento por predecir o explicar, de manera provisional, el fenómeno que se investigará para resolver la incertidumbre clínica. Debe ser expresada en forma de preposiciones y se debe asumir que no son necesariamente verdaderas ni que podrán ser comprobadas al final del estudio $(4,8)$. El razonamiento científico va de las teorías a las hipótesis y estas últimas se prueban en el mundo real; en este contexto, las hipótesis traducen una pregunta de investigación (problema de investigación) en una predicción precisa de los resultados esperados (4).

Una de las principales características de las hipótesis susceptibles de ser demostradas, es que enuncian la relación entre dos o más variables medibles, variable independiente y variable dependiente; en esta línea, el proceso de investigación clínica se orienta en la búsqueda de asociación entre diversas variables; la asociación encontrada puede ser real, producto del azar 0 afectado por la presencia de error sistemático. Para dilucidar este problema existen diversas estrategias estadísticas que permiten comprobar las hipótesis con una confiabilidad conocida. Estas herramientas permiten cuantificar hasta qué punto la variabilidad de la muestra en estudio (error aleatorio) es responsable de los resultados obtenidos. En investigación clínica, el riesgo de error aleatorio (error tipo I) máximo aceptado es $5 \%$, es decir, debe existir menos de un $5 \%$ de probabilidad de que los resultados obtenidos sean estadísticamente significativo cuando realmente no lo son, en la población en estudio (9).

\section{DISEÑOS DE INVESTIGACIÓN}

El diseño de investigación corresponde al plan o estrategia concebida para obtener la información que se desea. En el paradigma de investigación cuantitativa, los diseños se pueden utilizar para analizar la certeza de las hipótesis formuladas en un contexto en particular (diseños observacionales analíticos 0 experimentales) o para aportar evidencia respecto de los lineamientos de un tema de investigación en desarrollo cuando no es posible comprobar una hipótesis (diseños observacionales descriptivos) (10). A continuación se describen los diseños de investigación más empleados en investigación clínica:

\section{- Diseños observacionales}

Son aquellos donde el investigador se mantiene al margen de los hechos que ocurren. En estos diseños se debe considerar si la medición será única (estudio transversal) o a lo largo de un período de tiempo (estudio longitudinal). Adicionalmente, se debe decidir si el estudio se centrará en hechos del pasado (estudios retrospectivos) 0 en hechos del futuro (estudios prospectivos). Cabe destacar que la cantidad de mediciones y el 
carácter temporal del diseño corresponde a una característica y no a tipos de diseño en particular (10-12).

Los diseños observacionales pueden ser de dos tipos, descriptivos y analíticos:

Estudios observacionales descriptivos: están constituidos por los reportes de casos (reporte de 1 a 10 casos), series de casos (reporte de más de 10 casos), estudios de corte transversal (estudio donde todas las mediciones se realizan en una ocasión, sin periodo de seguimiento cuya medida de frecuencia susceptible de ser obtenida es la prevalencia) y estudios correlacionales (estudios poblacionales donde se establece el grado de relación existente entre dos o más variables) (10).

Estudios observacionales analíticos: están constituidos por los estudios de casos y controles (estudio retrospectivo basado en el análisis comparativo de un grupo de sujetos con la enfermedad 0 evento de interés [casos], con un grupo sin la enfermedad 0 evento de interés [controles], donde se evalúa si algún factor en estudio es distinto en uno u otro grupo, cuya medida de asociación es el odds ratio); estudios de cohorte (estudio longitudinal donde los grupos son clasificados según el factor de exposición [0 riesgo] y son seguidos hasta la aparición del evento de interés, cuya medida de frecuencia es la incidencia y de medida de asociación es el riesgo relativo); estudios de pruebas diagnósticas (donde se evalúa el rendimiento de una prueba diagnóstica en comparación a un estándar de referencia, cuyos parámetros evaluados son sensibilidad, especificidad, valores predictivos y likelihood ratio) y revisiones sistemáticas (donde se realiza una síntesis de la evidencia disponible y se obtiene una magnitud de resultado que equivale a una media ponderada según la calidad metodológica de los estudios primarios) (11-14).

\section{- Diseños experimentales}

Son aquellos donde el investigador interviene modificando la variable independiente y midiendo el impacto sobre las variables dependientes. En este contexto, el ensayo clínico constituye al representante por excelencia, gracias a que posee características que lo transforman en el diseño que proporciona uno de los más altos niveles de evidencia (estudio de cohortes prospectiva, experimental, con asignación aleatoria y enmascaramiento en los cuales el investigador manipula la variable predictiva, observando posteriormente el efecto en el grupo experimental y control). Adicionalmente existen los estudios cuasi experimentales (carecen de asignación aleatoria y enmascaramiento) y los diseños pre experimentales (antes y después) (15). En la tabla 1, se muestran los diseños de investigación más empleados en investigación clínica.
Tabla $\mathbf{N}^{0} \mathbf{1}$. Diseños de investigación más empleados en investigación clínica.

\begin{tabular}{|c|c|}
\hline $\begin{array}{c}\text { Diseños } \\
\text { Observacionales } \\
\text { Descriptivos }\end{array}$ & Característica \\
\hline Reportes de casos & - Reporte de menos de 10 casos. \\
\hline Series de casos & - Reporte de más de 10 casos. \\
\hline Corte transversal & $\begin{array}{l}\text { - Las variables son medidas en un momento } \\
\text { determinado, sin periodos de seguimiento. } \\
\text { - Permiten el cálculo de prevalencia. }\end{array}$ \\
\hline Correlacional & $\begin{array}{l}\text { - Evalúan el grado de correlación entre dos } \\
\text { variables cuantitativas. } \\
\text { - Los métodos estadísticos más empleados son el } \\
\text { coeficiente de correlación de Pearson (cuando la } \\
\text { distribución es normal) y coeficiente Rho de } \\
\text { Spearman (cuando la distribución no es normal). }\end{array}$ \\
\hline $\begin{array}{l}\text { Diseños } \\
\text { Observacionales } \\
\text { Analísticos }\end{array}$ & Característica \\
\hline Casos y controles & $\begin{array}{c}\text { • Estudio retrospectivo. } \\
\text { - Se evalúa en los casos y los controles la } \\
\text { presencia de factores de riesgo vinculados } \\
\text { a la enfermedad. } \\
\text { - La medida de asociación es el odds ratio (OR). }\end{array}$ \\
\hline Cohortes & $\begin{array}{l}\text { - Los grupos se clasifican según factor de } \\
\text { exposición y se evalúa de manera prospectiva } \\
\text { (o retrospectiva) la presencia de la enfermedad } \\
\text { en ambos grupos. } \\
\text { - La medida de frecuencia es la incidencia. } \\
\text { - La medida de asociación es el riesgo } \\
\text { relativo (RR). }\end{array}$ \\
\hline $\begin{array}{l}\text { Pruebas } \\
\text { diagnósticas }\end{array}$ & $\begin{array}{l}\text { - Evalúan el rendimiento de una prueba } \\
\text { diagnóstica al compararlo con el patrón de } \\
\text { referencia. } \\
\text { - El rendimiento diagnóstico se evalúa en función } \\
\text { de la sensibilidad, especificidad, valores } \\
\text { predictivos y cocientes de verosimilitud. } \\
\text { - Es posible expresar gráficamente el rendimiento } \\
\text { de una prueba diagnóstica a través de las } \\
\text { curvas ROC. }\end{array}$ \\
\hline $\begin{array}{l}\text { Revisiones } \\
\text { sistemáticas }\end{array}$ & $\begin{array}{l}\text { - Equivalen a una síntesis de la } \\
\text { evidencia publicada. } \\
\text { - Pueden ser revisiones cuantitativas } \\
\text { (meta análisis) o cualitativas. } \\
\text { - A través del proceso meta analítico es posible } \\
\text { obtener la magnitud del efecto, que equivale un } \\
\text { promedio ponderado según la calidad metodológica } \\
\text { de los artículos primarios. Permitiendo incrementar } \\
\text { notablemente la validez externa de los resultados. }\end{array}$ \\
\hline $\begin{array}{c}\text { Diseños } \\
\text { Experimental }\end{array}$ & Característica \\
\hline Ensayo clínico & $\begin{array}{c}\text { - Estudio de carácter prospectivo. } \\
\text { - Los grupos control y experimental se definen } \\
\text { por asignación aleatoria. } \\
\text { - Existe enmascaramiento (evaluador, sujeto de } \\
\text { estudio, quién aplica la intervención). } \\
\text { - Debe existir un seguimiento establecido y las } \\
\text { pérdidas no deben superar el 20\%. } \\
\text { - Se debe realizar análisis interno y análisis } \\
\text { por intención de tratar. } \\
\text { - Medidas de asociación: RR, NNT } \\
n^{\circ} \text { necesario para tratar), etc. }\end{array}$ \\
\hline
\end{tabular}




\section{CONCLUSIÓN}

El proceso de investigación nace en el momento en que la idea se transforma en un problema, para lo cual abordarlo desde la perspectiva de sus características principales, contexto e importancia, permitirá formular una pregunta de investigación que represente el sustento base del proceso. Entender la investigación como un proceso continuo, con etapas claramente establecidas da la posibilidad de ampliar el paradigma cuantitativo más allá de un mero análisis de una planilla de datos. En este sentido, las etapas iniciales abordadas en el presente texto, proporcionan al proceso un marco metodológico que contribuirá de manera significativa a la veracidad de los resultados obtenidos.

\section{REFERENCIAS}

1. Ries AL. Pulmonary rehabilitation: summary of an evidence-based guideline. Respir Care 2008; 53:1203-7

2. Sackett DL, Rosenberg WM, Gray JA, Haynes RB, Richardson WS. Evidence based medicine: what it is and what it isn't. BMJ 1996; $312: 71-2$

3. Rada G, Andrade M, Leyton V, Pacheco C, Ramos E. Búsqueda de información en medicina basada en evidencia. Rev Med Chile 2004; 132: 253-259

4. Hungler B, Polit D. Investigación científica en ciencias de la salud, McGraw-Hill Interamericana, México, 2000.

5. Centro Cochrane Iberoamericano, traductores. Manual Cochrane de
Revisiones Sistemáticas de Intervenciones, versión 5.1.0 [actualizada en marzo de 2011] [Internet]. Barcelona: Centro Cochrane Iberoamericano; 2012. Disponible en http://www.cochrane.es/?q=es/node/269.

6. Claro J, Lustig N, Soto M, Rada G. El primer paso: la pregunta clínica. Rev Med Chile 2012; 140: 1067 - 1072

7. Manterola C, Zavando D. Grupo Mincir. Cómo interpretar los "Niveles de Evidencia" en los diferentes escenarios clínicos. Rev Chil Cir 2009; 61: $587-600$

8. Manterola C, Otzen T. Por qué investigar y cómo conducir una investigación. Int J Morphol 2013; 31: 1498 - 1504

9. Manterola C, Pineda V. Grupo MINCIR. El valor de "p" y la "significancia estadística". Aspectos generales y su valor en la práctica clínica. Rev Chil Cir 2008; 60: 86-89

10. Manterola C. Estrategias de investigación. Diseños observacionales $1^{\circ}$ parte. Estudios descriptivos. Rev Chil Cir 2001; 53: 229 - 233

11. Manterola C, Muñoz S. Estrategias de investigación. Diseños observacionales $2^{\circ}$ parte. Estudios de casos y controles. Rev Chil Cir 2001; 53: $316-320$

12. Manterola C, Muñoz S, Bustos L. Estrategias de investigación. Diseños observacionales $3^{\circ}$ parte. Estudios de cohortes. Rev Chil Cir 2001; 53: $410-414$

13. Manterola C, Astudillo P, Arias E, Claros N. Revisiones sistemáticas de la literatura. Qué se debe saber acerca de ellas. Cir Esp 2013;91:149155

14. Letelier L, Manríquez J, Rada G. Revisiones sistemáticas y metaanálisis: ¿son la mejor evidencia?. Rev Med Chile 2005; 133: 246 $-249$

15. Hernández R, Fernández C, Baptista P. Metodología de la investigación, McGraw-Hill Interamericana, 4 Edición, México D.F, 2006.

El autor declara no tener conflicto de intereses. 